\title{
Manejo conservador en diverticulitis aguda perforada con aire extraluminal
}

\author{
Macarena Paz Fernández A., ${ }^{1,2}$ \\ TRABAJO DE INGRESO A LA SOCIEDAD CHILENA DE COLOPROCTOLOGÍA
}

\section{Conservative management of perforated diverticulitis with extraluminal air}

Background: The conservative management of perforated diverticulitis with extraluminal air (PDA) is controversial. In selected hospitalized patients may offer less morbidity and mortality and avoid an ostomy. Aim: To describe its clinical and imagenologic characteristics, the need for surgical procedure and morbimortality of patients with perforated diverticulitis with extraluminal air in conservative management. Materials and Methods: Retrospective descriptive study of hemodynamically stable hospitalized patients with a PDA diagnosis without evidence of peritonitis during the years 2009 and 2015. Results: 162 patients were admitted with the diagnosis of acute diverticulitis. $53.1 \%$ of cases were acute complicated diverticulitis, 43\% (37 cases) were PDA. The average age was 59.6 years and most of them women $(54.1 \%)$. The most common clinical manifestations were abdominal pain $(97.3 \%)$, peritoneal signs $(59.5 \%)$ and fever $(40.5 \%)$. The abdomen and pelvic computerized axial tomography scan showed extra-colonic bubbles in $78.4 \%$ and pneumoperitoneum in $21.6 \%$. The conservative management was successful in $87.8 \%$ of cases. After a 71.2-month follow-up, the recurrence was $28.1 \%$ and $31.3 \%$ had an elective sigmoidectomy. 5 cases did not respond to medical treatment, one of them resulting in death. Conclusion: The conservative management of PDA is a valid treatment option with a high degree of success in the selected sample of hospitalized patients. The decision of having emergency surgery and elective sigmoidectomy post DPA should be evaluated individually.

Key words: perforated diverticulitis; complicated diverticulitis; extraluminal air; nonoperative management.

\section{Resumen}

Introducción: El tratamiento óptimo de la diverticulitis perforada con aire extraluminal (DPA) es materia de debate. El manejo conservador es controversial; en casos seleccionados puede ofrecer menor morbimortalidad y evitar una ostomía. Objetivo: Describir las características clínicas, imagenológicas, necesidad de intervención quirúrgica y morbimortalidad de pacientes con DPA tratados con manejo conservador. Materiales y Método: Estudio descriptivo retrospectivo de pacientes hospitalizados con diagnóstico de DPA hemodinámicamente estables sin evidencia de peritonitis entre los años 2009 y 2015. Resultados: Se hospitalizaron 162 pacientes con diagnóstico de diverticulitis aguda (DA), el 53,1\% fueron diverticulitis agudas complicadas (DC), de las cuales el 43\% (37 casos) eran DPA, la edad promedio fue 59,6 años, la mayoría eran mujeres $(54,1 \%)$. Las manifestaciones clínicas más comunes fueron dolor abdominal $(97,3 \%)$, signos peritoneales $(59,5 \%)$ y fiebre $(40,5 \%)$. La tomografía axial computada de abdomen y pelvis (TC AP) mostró burbujas extracolónicas en el 78,4\% y neumoperitoneo en el 21,6\%. El manejo conservador fue exitoso en el 87,8\%. Durante el seguimiento de 71,2 meses la recurrencia fue $28,1 \%$ y el $31,3 \%$ se realizó sigmoidectomía electiva. La falla del tratamiento médico se presentó en 5 casos (12,2\%), uno de los cuales fallece. Conclusión: el manejo conservador de la DPA es una alternativa válida de tratamiento con alto porcentaje de éxito en pacientes hospitalizados seleccionados. La decisión de cirugía de urgencia y de sigmoidectomía electiva posterior a una DPA tratada médicamente debe ser individualizada.

Palabras clave: diverticulitis complicada; diverticulitis perforada; aire extraluminal.
'Unidad de Coloproctología, Departamento de Cirugía, Hospital Militar. Santiago, Chile.

2Facultad de Medicina, Universidad de los Andes. Santiago, Chile.

Recibido el 17 de agosto de 2019 y aceptado para publicación el 4 de septiembre de 2019.

Correspondencia a: Dra. Macarena Paz Fernández A.

contacto@

dramacarenafernandez.cl 


\section{Introducción}

La enfermedad diverticular colónica ha aumentado en un $26 \%$ su incidencia durante la última década $^{1}$. La mayoría son diverticulitis simples (DS) con adecuada respuesta a tratamiento médico ambulatorio. Sin embargo, el 15\%-25\% de estos pacientes desarrollará durante su evolución una diverticulitis complicada (DC) incluyendo abscesos, fístulas, estenosis, peritonitis o perforación con aire extraluminal ${ }^{2,3}$; de hecho, la diverticulitis aguda (DA) es la causa más frecuente de perforación colónica, abarcando el $60 \%$ de todos los casos ${ }^{4}$.

El tratamiento óptimo de la diverticulitis aguda complicada es materia de debate, si bien la mayoría de las guías clínicas recomienda la sigmoidectomía de urgencia, la tendencia actual es evitar la emergencia quirúrgica a través de terapias menos invasivas con un claro beneficio en morbimortalidad ${ }^{5-9}$. En este contexto publicaciones recientes plantean que el manejo no quirúrgico de pacientes con diverticulitis perforada con aire extraluminal (DPA) es una alternativa terapéutica controversial, que en casos seleccionados puede ofrecer buenos resultados y evitar la necesidad de una ostomía ${ }^{10-15}$.

El objetivo de este trabajo es describir las características clínicas, imagenológicas, necesidad de intervención quirúrgica, morbimortalidad y recurrencia de pacientes con diagnóstico de DPA tratados con manejo conservador.

\section{Materiales y Método}

Se realizó un estudio descriptivo retrospectivo de pacientes hospitalizados con diagnóstico de DPA en un hospital institucional de nivel terciario durante los años 2009 y 2015. Todos los pacientes fueron evaluados con una TC AP al ingreso. La DPA se definió como la existencia de una DA asociada a la presencia de burbujas extraluminales y/o neumoperitoneo en la TC AP.

Los casos de DPA seleccionados para manejo conservador cumplieron con los siguientes criterios de inclusión:

1) Pacientes hospitalizados con diagnóstico de DPA.

2) Líquido libre intraabdominal ausente o laminar en TC AP al ingreso.

3) Estabilidad hemodinámica (presión arterial media mayor a $70 \mathrm{sin}$ apoyo de drogas vasoactivas).

4) Dolor abdominal controlado (igual o menor a 3 en escala visual análoga).

El manejo médico conservador consistió en reposo digestivo y administración parenteral de hidratación, antibióticos de amplio espectro y analgesia más antiinflamatorios en bomba de infusión continua. En caso de evolución favorable posterior a las $24-48 \mathrm{~h}$ de hospitalización, se indica realimentación progresiva y se evalúa la tolerancia a la administración de antibióticos vía oral y analgesia horaria; si existe respuesta clínica satisfactoria el paciente es dado de alta con indicación de control clínico ambulatorio posterior a completar 10-14 días de tratamiento antibiótico en domicilio.

Se consideró falla del manejo conservador las siguientes situaciones clínicas:

1) Inestabilidad hemodinámica durante hospitalización.

2) Mantención del cuadro séptico abdominal (dolor no controlado, fiebre, taquicardia, parámetros inflamatorios estacionarios).

3) Progresión imagenológica de la DPA con aparición de líquido abdominal de mayor cuantía sugerente de peritonitis y/o la aparición de absceso intraabdominal durante la terapia antibiótica.

El número total de pacientes hospitalizados con diagnóstico de DA se obtuvo del registro diario de ingresos al Servicio de Cirugía durante el período descrito. Los datos fueron obtenidos de la revisión de la ficha clínica de cada paciente, siguiendo las normas establecidas por nuestro centro asistencial. La información recopilada fue sometida a un análisis estadístico univariado. Los resultados son expresados en porcentajes.

\section{Resultados}

Entre abril del año 2009 y julio del año 2015 se hospitalizaron 162 pacientes con diagnóstico de DA.

El 53,1\% (86 pacientes) ingresó con diagnóstico de DC, de este grupo el $43 \%$ (37 pacientes) eran DPA; el 45,4\% (39 casos) eran abscesos intraabdominales (con o sin aire extraluminal asociado); el $9,3 \%$ ( 8 casos) tenía una peritonitis difusa al momento del ingreso, razón por la cual fueron directamente a cirugía de urgencia evidenciado una peritonitis de origen diverticular Hinchey III o IV en el intraoperatorio (se efectuaron 4 aseos peritoneales más drenajes, 2 sigmoidectomías abiertas con anastomosis primaria y 2 operaciones de Hartmann). Hubo un caso de pileflebitis y otro paciente con una neumatosis portal extensa, dos complicaciones infrecuentes secundarias al episodio diverticular agudo, ambos con adecuada respuesta a manejo médico.

El $46,9 \%$ restante (76 pacientes) eran DS con 
indicación de hospitalización, dada principalmente por dolor abdominal persistente, signos peritoneales, mala tolerancia oral y/o descompensación de comorbilidades.

De los pacientes con DPA, la edad promedio fue 59,6 años (21-92 años) y el 54,1\% eran mujeres. Las comorbilidades más comunes en este grupo fueron hipertensión arterial $(40,5 \%)$, diabetes mellitus o insulinorresistencia (24,3\%), dislipidemia (16,2\%) e hipotiroidismo $(13,5 \%)$. Sólo 2 pacientes eran inmunosuprimidos con tratamiento corticoidal crónico. El 18,9\% (7 pacientes) tenía el antecedente de DS.

El cuadro clínico previo a consultar fue de 2,8 días promedio de evolución, caracterizado por dolor abdominal en $97,3 \%$, fiebre en $45,9 \%$, náuseas y/o vómitos en 37,8\% y diarrea en el 18,9\% de los casos. Al ingreso todos los pacientes estaban hemodinámicamente estables, con taquicardia el $24,3 \%$, fiebre el $40,5 \%$, signos peritoneales $59,5 \%$, leucocitosis en 70,3\%, leucopenia 5,4\% ( 2 casos), y proteína $\mathrm{C}$ reactiva mayor a $100 \mathrm{mg} / \mathrm{L}$ en $43,2 \%$.

La TC AP mostró en todos los casos de DPA presencia de divertículos colónicos, engrosamiento parietal y aumento de la densidad del tejido graso adyacente; en el 78,4\% (29 pacientes) evidenció burbujas extraluminales pericolónicas y en el $21,6 \%$ restante ( 8 pacientes) neumoperitoneo distante al colon, con o sin burbujas pericolónicas asociadas.

El manejo conservador fue exitoso en el 86,5\% de las DPA (32 casos), con remisión total del cuadro clínico durante una estadía hospitalaria promedio de 4,3 días (2-12 días) y tratamiento antibiótico por 14 días promedio de duración que se logró completar de forma ambulatoria en todos los casos. Todos estos pacientes fueron estudiados con una colonoscopía o enema baritado 6 a 8 semanas posterior al episodio de DA descartando cáncer colorrectal. Durante una mediana de seguimiento de 71 meses (extremos 38-103) sólo el 31,3\% (10 pacientes) fue sometido a una sigmoidectomía laparoscópica electiva sin incidentes ni morbimortalidad asociada. El $28,1 \%$ (9 casos) ha presentado nuevos episodios de DS sin necesidad de cirugía de urgencia.

El fracaso del manejo conservador se presentó en 5 pacientes $(13,5 \%)$. Una paciente con múltiples comorbildades y necesidad de tratamiento corticoidal crónico por nefropatía autoinmune evolucionó con una colección pericolónica sin respuesta a manejo conservador y sin ventana adecuada para instalación de drenaje percutáneo. Se efectuó una sigmoidectomía abierta con anastomosis primaria, evolucionando satisfactoriamente. Otro paciente mantuvo parámetros inflamatorios elevados y colección pericolónica persistente en ángulo esplénico en TC AP de control pese a punción percutánea. Se realizó resección y anastomosis primaria sin incidentes, confirmando posteriormente el diagnóstico de cáncer colorrectal. Dos pacientes con buena respuesta inicial a manejo conservador, posterior al alta, consultan por dolor abdominal y fiebre; reingresan con el diagnóstico de colección intraabdominal, ambos se trataron con drenaje percutáneo guiado por TC AP y tratamiento antibiótico prolongado. El quinto y último caso se trata de una paciente octogenaria portadora de una demencia avanzada, a las $48 \mathrm{~h}$ de manejo conservador presenta quiebre clínico y aparición de líquido libre moderada cuantía en TC AP de control. Se decide realizar una operación de Hartmann frente a una peritonitis purulenta difusa. La paciente evoluciona con falla multiorgánica, falleciendo al séptimo día postoperatorio, constituyendo el único caso de cirugía de urgencia, ostomía y mortalidad de nuestra serie $(2,7 \%)$.

\section{Discusión}

La prevalencia de la enfermedad diverticular ha aumentado considerablemente durante el último siglo, siendo actualmente uno de los cinco desórdenes gastrointestinales más costosos a nivel mundial ${ }^{4,16}$. La perforación asociada a una DA representa el 75\% de las urgencias diverticulares que requieren cirugía $^{18}$, con una tasa de morbilidad del $40 \%-44 \%{ }^{17,18}$ y una tasa de mortalidad del 4,4-23,7\% ${ }^{17-20}$.

Existe común acuerdo en que los pacientes con una diverticulitis perforada asociada a una sepsis abdominal persistente, mala respuesta a manejo conservador o con evidencia clínica de una peritonitis diverticular al ingreso o durante la hospitalización se benefician de una intervención quirúrgica precoz, con la posibilidad de realizar una operación de Hartamann $^{21-25}$, resección colónica más anastomosis primaria con o sin ostomía de protección ${ }^{24-28}$ o lavado peritoneal laparoscópico ${ }^{29-33}$ según la situación clínica de cada paciente, los medios disponibles y la experiencia del equipo quirúrgico tratante, tomando en cuenta que el $30 \%-40 \%$ de los pacientes ostomizados jamás se reconstituye, lo que genera una importante alteración de la calidad de vida, morbilidad asociada a la ostomía y altos costos de mantención ${ }^{21-25}$.

Considerando esta realidad, la tendencia actual es un abordaje menos invasivo para la DC; varios autores y algunas guías clínicas dan cuenta de esta afirmación $^{5-9}$. Una situación especial constituye la DA perforada asociada a neumoperitoneo y/o burbujas extracolónicas. La presencia de aire extraluminal es 
un signo objetivo y fácil de identificar en la TC AP, que demuestra que se ha producido una perforación colónica; no obstante la verdadera importancia de este hallazgo aún no se ha determinado. Algunos autores lo consideran como un signo de peritonitis difusa en curso mientras otros no lo mencionan en el proceso de la toma de decisiones ${ }^{34,35}$.

Históricamente se ha utilizado la Clasificación de Hinchey ${ }^{36}$ extrapolada a los hallazgos imagenológicos para definir conducta terapéutica. Esta clasificación ha tenido modificaciones en el tiempo y recién durante la última década se incorpora la presencia de aire extraluminal, la localización y cuantía de éste, además de precisar la ubicación, número y tamaño de los abscesos intraabdominales, con el fin de definir el tratamiento óptimo con la mayor información disponible $^{37-39}$ (Tabla 1).

Los datos obtenidos en esta serie muestran que la mayoría de los pacientes con DPA puede recibir un tratamiento no quirúrgico con una tasa de éxito del $86,5 \%$, con un $28,1 \%$ de recurrencia donde todos los casos fueron nuevamente susceptibles a manejo médico con respuesta favorable. Nuestros datos son consistentes con otras series publicadas, apoyando la visión general de un manejo menos invasivo para la DC tanto en la indicación de cirugía de urgencia como en la intervención quirúrgica electiva, esta última realizada sólo en el $31,3 \%$ de nuestros ca$\operatorname{sos}^{10-13}$ (Tabla 2).

Dentro de los factores implicados en la falla del manejo conservador están la clasificación ASA III-IV, leucocitosis $>20.000$ por $\mathrm{mm}^{3}$, colección intraabdominal concomitante y gran volumen de aire extraluminal en la TC AP ( $>2 \mathrm{~cm})$. La ubicación del aire extracolónico como factor de riesgo independiente para falla del manejo no quirúrgico es controversial, existen estudios que muestran al aire pericolónico y otros que indican al aire distal como factor involucrado en un tratamiento conservador no exitoso $^{10-13}$. Es difícil definir si estos factores tienen relevancia en el porcentaje de fracaso del manejo médico de nuestra serie dado el escaso número de pacientes ( 5 casos).

Existe evidencia suficiente para considerar a los pacientes inmunosuprimidos como grupo de alto riesgo, con tasa significativamente más alta de perforación libre, cirugía de urgencia y mortalidad postoperatoria, por lo tanto, debe considerarse un abordaje quirúrgico precoz en caso de DC independiente de que no exista evidencia de peritonitis o compromiso hemodinámico, situación presente en una de nuestras pacientes con falla del manejo conservador ${ }^{14}$.

La incidencia de cáncer de colon en pacientes con

Tabla 1. Clasificación de la diverticulitis aguda complicada

\begin{tabular}{|c|c|c|c|c|}
\hline & $\begin{array}{c}\text { Clasificación de Hinchey } \\
\left(\mathbf{( 1 9 7 8}^{36}\right.\end{array}$ & $\begin{array}{c}\text { Clasificación de Buckley } \\
(2004)^{37}\end{array}$ & Criterios de Siewert (2006) & $\begin{array}{l}\text { Clasificación de Neff } \\
\text { modificada }(2013)^{39}\end{array}$ \\
\hline ETAPA 0 & & & & $\begin{array}{l}\text { DA no complicada } \\
\text { Engrosamiento parietal, } \\
\text { aumento de la densidad de } \\
\text { tejido graso adyacente }\end{array}$ \\
\hline ETAPA I & Absceso pericolónico & $\begin{array}{l}\text { DA leve } \\
\text { Engrosamiento parietal y } \\
\text { cambios de la grasa pericólica }\end{array}$ & $\begin{array}{l}\text { Aire libre localizado } \\
\text { (pericolónico) sin absceso }\end{array}$ & $\begin{array}{l}\text { DA complicada localizada } \\
\text { Etapa IA: burbujas pericólicas } \\
\text { Etapa IB: absceso }<4 \mathrm{~cm}\end{array}$ \\
\hline ETAPA II & Absceso pélvico & $\begin{array}{l}\text { DA moderada } \\
\text { Grosor pared }>3 \mathrm{~mm} \text {, con } \\
\text { flegmón o formación de } \\
\text { pequeños abscesos }\end{array}$ & $\begin{array}{l}\text { Colección pequeña }<2 \mathrm{~cm} \\
\text { con aire libre distante o } \\
\text { absceso }<4 \mathrm{~cm}\end{array}$ & $\begin{array}{l}\text { DA complicada } \\
\text { Absceso pélvico }>4 \mathrm{~cm}\end{array}$ \\
\hline ETAPA III & $\begin{array}{l}\text { Peritonitis difusa (ruptura de } \\
\text { absceso pericolónico/pélvico) }\end{array}$ & $\begin{array}{l}\text { DA severa } \\
\text { Grosor pared }>5 \mathrm{~mm} \text {, } \\
\text { perforación con aire libre } \\
\text { subdiafragmático o absceso } \\
>5 \mathrm{~cm}\end{array}$ & $\begin{array}{l}\text { Colección grande }>2 \mathrm{~cm} \text { con } \\
\text { aire libre distante o } \\
\text { Absceso }>4 \mathrm{~cm}\end{array}$ & $\begin{array}{l}\text { DA complicada } \\
\text { Absceso distante en cavidad } \\
\text { abdominal }\end{array}$ \\
\hline ETAPA IV & $\begin{array}{l}\text { Peritonitis fecaloídea } \\
\text { (macroperforación de un } \\
\text { divertículo) }\end{array}$ & & $\begin{array}{l}\text { Aire libre con líquido intra- } \\
\text { abdominal no loculado } \\
\text { (peritonitis fecaloídea) }\end{array}$ & $\begin{array}{l}\text { DA complicada } \\
\text { Neumoperitoneo abundante } \\
\text { y/o líquido libre intra- } \\
\text { abdominal }\end{array}$ \\
\hline
\end{tabular}


Tabla 2. Manejo conservador diverticulitis perforada con aire extraluminal

\begin{tabular}{|lccccccccc|}
\hline & $\mathbf{n}$ & $\begin{array}{c}\text { Aire } \\
\text { distante }\end{array}$ & $\begin{array}{c}\text { Aire } \\
\text { pericolónico }\end{array}$ & Éxito & Fracaso & $\begin{array}{c}\text { Estadía } \\
\text { (días) }\end{array}$ & $\begin{array}{c}\text { Cirugía } \\
\text { electiva }\end{array}$ & Mortalidad & Recurrencia \\
$\begin{array}{l}\text { Dharmarajan } \\
\text { (USA, 2011) }\end{array}$ & 131 & $112^{*}$ & 19 & $124(95 \%)$ & $7(5 \%)$ & $5,7-10,7$ & $75(57 \%)$ & $1(0,7 \%)$ & No descrito \\
$\begin{array}{l}\text { Costi1 } \\
\text { (Italia, 2012) }\end{array}$ & 39 & 8 & 31 & $36(92 \%)$ & $3(8 \%)$ & 8,1 & $17(47 \%)$ & NO & $16,1 \%$ \\
$\begin{array}{l}\text { Sallinen'12 } \\
\text { (Finlandia, 2014) }\end{array}$ & 125 & 43 & 81 & $105(84 \%)$ & $20(16 \%)$ & $3-23$ & No descrito & $1(0,8 \%)$ & No descrito \\
$\begin{array}{l}\text { Titos-García' } \\
\text { (España 2017) }\end{array}$ & 64 & 13 & 51 & $54(84 \%)$ & $10(16 \%)$ & $11,6-13,4$ & No descrito & $1(1,6 \%)$ & $20,4 \%$ \\
$\begin{array}{l}\text { Hospital Militar } \\
\text { (Chile, 2018) }\end{array}$ & 37 & 8 & 29 & $32(86,5 \%)$ & $5(13,2 \%)$ & 4,3 & $10(31,3 \%)$ & $1(2,7 \%)$ & $28,1 \%$ \\
\hline
\end{tabular}

*Incluye en esta categoría pacientes con abscesos diverticulares.

DA es cercana al 2\%; no obstante, la sobreposición diagnóstica de DA y neoplasia de colon se describe hasta en un $50 \%$ de las TC AP durante la evaluación de un episodio diverticular agudo. La longitud del colon comprometido $<10 \mathrm{~cm}$, adenopatías y la evidencia de complicación (absceso, fístula, perforación) son hallazgos tomográficos que se relaciona con mayor frecuencia a neoplasia colónica en pacientes con diagnóstico inicial de $\mathrm{DA}^{40}$. En este grupo es importante recalcar la importancia de la colonoscopía 4-6 semanas después de terminar el tratamiento antibiótico, siempre que la condición del paciente lo permita ${ }^{8-9}$, lo cual no fue factible en uno de nuestros casos por presentar sepsis abdominal persistente, por lo cual el diagnóstico definitivo fue postoperatorio.

La aparición de colecciones abdominales durante el tratamiento antibiótico ambulatorio es parte de la morbilidad esperada incluso en casos de DS. Varios autores no lo consideran fracaso del manejo conservador ya que la inmensa mayoría no requiere cirugía, basta con una punción percutánea guiada por TC AP y mantención del tratamiento antibiótico $^{10-13}$, tal como sucedió en los 2 casos descritos que evolucionaron con un absceso diverticular posterior a una respuesta inicial favorable.

La mortalidad descrita en nuestro trabajo concuerda con lo publicado recientemente, donde no supera un caso en cada serie (Tabla 2). Estimamos que este protocolo de manejo no quirúrgico en DPA debe ser llevado con mayor cautela en pacientes añosos con compromiso cualitativo de conciencia, ya que pueden existir limitaciones en la obtención correcta de la anamnesis y en la exploración física objetiva.

\section{Conclusiones}

El manejo conservador de la DPA es una opción válida y eficaz en pacientes seleccionados; si bien la mayoría tiene un comportamiento benigno similar a una DS, aún no existe evidencia suficiente que justifique un manejo ambulatorio seguro, ya que un pequeño número de pacientes progresa a un cuadro séptico grave, asociado a complicaciones y mortalidad.

La literatura médica disponible ha cuestionado la mayor parte de los paradigmas que han regido el manejo clínico de la DC y la evidencia existente aún no es concluyente en varios tópicos. El problema principal radica en reconocer dentro de un grupo heterogéneo de pacientes portadores de una DPA, la severidad de cada uno identificando adecuadamente qué casos se benefician del tratamiento conservador y qué casos requieren un manejo quirúrgico inicial.

En este sentido, consideramos importante actualizar la clasificación clínica radiológica de la DC considerando la presencia y características del aire extracolónico, con el fin de tomar una decisión terapéutica más precisa y poder comparar resultados de manera más objetiva en este grupo específico de pacientes.

Los pacientes tratados con éxito tienen un riesgo similar de recurrencia que los pacientes que presentan episodios sin complicaciones, por lo tanto, la indicación de sigmoidectomía electiva debería ser individualizada, considerando siempre en esta decisión las características del paciente, la necesidad de excluir patología subyacente, la experiencia del equipo quirúrgico y los recursos disponibles en cada centro hospitalario. 


\section{Agradecimientos}

A los Dres. Leonardo Espíndola Silva, Andrés Larach Kattán y Joaquín Irarrázaval Espinosa.

\section{Responsabilidades éticas}

Protección de personas y animales. Los au- tores declaran que para esta investigación no se han realizado experimentos en seres humanos ni en animales.

Confidencialidad de los datos. Los autores declaran que en este artículo no aparecen datos de pacientes.

Conflictos de interés: no hay.

\section{Bibliografía}

1. Etzioni D, Chiu V, Cannom R, Burchette R, Haigh P, Abbas M. Outpatient treatment of acute diverticulitis: rates and predictors of failure. Dis Colon Rectum 2010;53:861-5.

2. Vermeulen J, Gosselink M, Hop W, Lange J, Coene P, van der Harst E, et al. Hospital mortality after emergency surgery for perforated diverticulitis. Ned Tijdschr Geneeskd. 2009;153:1209-14.

3. Forzard J, Armitage N, Schofield J, Jones O. Association of Coloproctology of Great Britain and Ireland. ACPGBI position statement on elective resection for diverticulitis. Colorectal Dis. 2011;13 Suppl 3:1-11.

4. Etzioni D, Mack T, Beart R, Kaiser A. Diverticulitis in the United States: 19982005: Changing patterns of disease and treatment. Ann Surg. 2009;249:210-7.

5. Nally D, Kavanagh D. Current controversies in the management of diverticulitis: a review. Dig Surg. 2019;3:195-205.

6. Sartelli M, Catena F, Ansaloni L, Coccolini F, Griffihs E, Abu-Zidan F, et al. WSES guidelines for the management of acute left sided colonic diverticulitis in the emergency setting. World J of Emerg Surg. 2016;11:37. doi:10.1186/s13017016-0095-0.

7. Stollman N, Smalley W, Hirano I; AGA Institute Clinical Guidelines Committee. American gastroenterological association Institute Guideline on the management of acute diverticulitis. Gastroenterology 2015;149:1944-9.

8. Bugiantella W, Rondelli F, Longaroni M, Mariani E, Sanguinetti A, Avenia N. Left colon acute diverticulitis: an update on diagnosis, treatment and prevention. Int $\mathbf{J}$ Surg. 2015;13:157-64.
9. Regenbogen S, Hardiman K, Hendren S, Morris A. Surgery for diverticulitis in the $21^{\text {st }}$ century: a systematic review. JAMA Surg. 2014:149:292-302.

10. Dharmarajan S, Hunt S, Birnbaum E, Fleshman J, Mutch M. The efficacy of nonoperative management of acute complicated diverticulitis. Dis Colon Rectum 2011;54:663-71.

11. Costi R, Cauchy F, LeBian A, Honart J, Creuze N, Smadja C. Challenging a classic myth: pneumoperitoneum associated with diverticulitis is not an indication for open or laparoscopic emergency surgery in hemodynamically stable patients. A 10 -year experience with a nonoperative treatment. Surg Endosc. 2012;26:2061-71.

12. Sallinen V, Mentula P, Lappaniemi A. Nonoperative management of perforated diverticulitis with extraluminal air is safe and effective in selected patients. Dis Colon Rectum 2014;57:875-81

13. Titos A, Aranda J, Romacho L, González A, Cabrera I, Santoyo J. Nonoperative management of perforated acute diverticulitis with extraluminal air: results and risk factors of failure. Int $\mathrm{J}$ Colorectal Dis. 2017;32:1503-7.

14. Khalil H, Yoo J. Colorectal emergencies: perforated diverticulitis (operative and nonoperative management). J Gastrointest Surg. 2014;18:865-8.

15. Suárez J, Amoza S, Batlle $X$, Oronzo B, Balen E, Yarnoz C. Safety of nonoperative management after acute diverticulitis. Ann Coloproctol. 2014;30:216-21.

16. Hall J, Hammerich K, Roberts P. New paradigms in the management of diverticular disease. Curr Probl Surg. 2010;47:680-753.

17. Chapman J, Davies M, Wolff B, Dozois E, Tessier D, Harrington J, et al. Complicated diverticulitis: is it time to rethink the rules. Ann Surg. 2005;242:576-81.

18. Schwesinger W, Page C, Gaskill H, Steward R, Chopra S, Strodel W, et al. Operative management of diverticular emergencies: strategies and outcomes. Arch Surg. 2000;135:558-62.

19. Biondo S, Ramos E, Deiros M, Rague J, De Oca J, Moreno P, et al. Prognostic factors for mortality in left colonic peritonitis: a new scoring system. J am Coll Surg. 2000;191:635-42.

20. Chandra V, Nelson H, Larson D, Harrington J. Impact of primary resection on the outcome of patients with perforated diverticulitis. Arch Surg. 2004;139:12214.

21. Abbas S. Resection and primary anastomosis in acute complicated diverticulitis, a systematic review of the literature. Int J Colorectal Dis. 2007;22:351-7.

22. Maggard M, Zingmond D, O'Connell J, Ko C. What proportion of patients with an ostomy (for diverticulitis) get reversed? Am Surg. 2004;70:928-31.

23. Banerjee S, Leather A, Rennie J, Samano N, González J, Papagrigoriadis S. Feasibility and morbidity of reversal of Hartmann's. Colorectal Dis. 2005;7:454-9.

24. Gooszen A, Tollenaar R, Geelkerken R, Smeets H, Bemelman W, Schaardenburgh $\mathrm{P}$, et al. Prospective study of primary anastomosis following sigmoid resection for suspected acute complicated diverticular disease. Br J Surg. 2001;88:693-7.

25. Richter S, Lindemann W, Kollmar O, Pistorius G, Maurer C, Schilling M. One-stage sigmoid colon resection for perforated diverticulitis (Hinchey stages III and IV) World J Surg. 2006;30:102732.

26. Constantinidez V, Heriot A, Remzi F, 
Darzi A, Senapati A, Fazio V, et al. Operative strategies for diverticular peritonitis: a decisión analysis between primary resection and anastomosis versus Hartmann's procedures. Ann Surg. 2007;245:94-103.

27. Kreis M, Mueller M, Thasler W. Hartmann's procedure or primary anastomosis? Dig Dis. 2012;30:83-5.

28. Vermeulen J, Coene P, Hout N, Harst E, Gosselink M, Mannaerts G, et al. Restoration of bowel continuity after surgery for acute perforated diverticulitis. Should Hartmann's procedure be considered a one-stage procedure? Colorectal Dis. 2009;11:619-24.

29. Myers E, Hurley M, O'Sullivan G, Kavanagh D, Wilson I, Winter D. Laparoscopic peritoneal lavage for generalized peritonitis due to perforated diverticulitis. Br J Surg. 2008;95:97-101.

30. Franklin M, Portillo G, Treviño J, González J, Glass J. Long-term experience with the laparoscopic approach to perforated diverticulitis plus generalized peritonitis. World J Surg. 2008;32:150711.

31. Afshar S, Kurer M. Laparoscopic peritoneal lavage for perforated sigmoid diverticulitis. Colorrectal Dis. 2012;14:135-42.

32. White S, Frenkiel B, Martin P. A ten-year audit of perforated sigmoid diverticulitis: highlighting the outcomes of laparoscopic lavage. Dis Colon Rectum. 2010;53:153741.

33. Alamili M, Gögenur I, Rosenberg J. Acute complicated diverticulitis managed by laparoscopic lavage. Dis Colon Rectum 2009;52:1345-9.

34. Ritz J, Lehmann K, Loddenkemper C, Frericks B, Buhr H, Holmer C. Preoperative CT staging in sigmoid diverticulitis-does it correlate with intraoperative and histological findings? Langenbecks Arch Surg. 2010;395:100915.

35. Kaiser A, Jiang J, Lake J, Ault G, Artinyan A, González-Ruiz C, et al. The management of complicated diverticulitis and the role of computed tomography. Am J Gastroenterol. 2005;100:910-7.

36. Hinchey E, Schaal P, Richards G. Treatment of perforated diverticular disease of the colon. Adv Surg. 1978;12:85-109.

37. Buckley O, Geoghegan T, O'Riordain D, Lyborn I, Torreggiani W. Computed tomography in the imaging of colonic diverticulitis. Clin Radiol. 2004;59:97783.

38. Siewert B, Tye G, Kruskal J, Sosna J, Opelka F, Raptopoulos V, et al. Impact of CT-guided drainage in the treatment of diverticular abscesses: size matters. AJR Am J Roentgenol. 2006;186:680-6.

39. Mora L, Serra S, Serra X, Ballesteros E, Navarro S. Application of a modified Neff classification to patients with uncomplicated diverticulitis. Colorrectal Dis. 2013;15:1442-7.

40. Chintapalli K, Chopra S, Ghiatas A, Esola C, Fields S, Dodd G. Diverticulitis versus colon cancer: differentiation with helical CT findings. Radiology 1999;210:429-35.

\section{Informe Trabajo de ingreso a Sociedad Chilena de Coloproctología de la Dra. Macarena Fernández Albornoz}

\section{Análisis metodológico}

\section{Introducción}

Se hace un buen planteamiento del problema, es una enfermedad frecuente y relevante. El manejo puede ser conservador o quirúrgico, y la elección por uno u otro tratamiento, depende de muchos factores, aún en proceso de definir.

Clásicamente se ha definido a la DC como diverticulitis asociada con perforación, fístula, absceso, estenosis u obstrucción (ASCRS 2016). Ya en este grupo existe controversia en el enfrentamiento debido a la morbilidad e incluso mortalidad del tratamiento quirúrgico.

En esta línea es que se está buscando el mejor tratamiento, menos invasivo y acorde con el conocimiento cada vez más sólido de la historia natural de la enfermedad y desarrollo de la tecnología.

La Dra. Fernández nos presenta entonces un tema muy actual, al incorporar el aire libre como variable a considerar en clasificaciones de evaluación al ingreso por CT de la diverticulitis complicada; los trabajos que analizan como una opción de tratamiento diferente la sola presencia de aire libre en un paciente con DC y condiciones definidas son recientes.

Incluso en una publicación de Journal of Colorectal Disease de enero de 2018, en que un autor alemán realiza una revisión sistemática comparan do distintas guías de manejo de enfermedad diverticular, que incluye la ASCRS 2014, italiana, germana, danesa, holandesa, AGA 2015 y otras; en ninguna de ellas consideran como un grupo en particular al DPA. Ello da aún más valor a este trabajo de la Dra. Fernández al adelantarse en revisar sus resultados.

\section{Objetivo}

Describir características clínicas, imagenológicas, indicación quirúrgica, morbimortalidad y recurrencia postratamiento conservador. Neff et al., establece en 19895 grupos desde 0-4 (0: DA no complicada 4: neumoperitoneo con abundante líquido libre). Luego, el año 2013 el grupo español de Mora López la modifica agregando: el 1a neumo pericolónico en forma de burbujas, $1 \mathrm{~b}$ absceso pericólico, 2 absceso pélvico y 3 absceso o aire libre a distancia. En 1997 Sher (Hinchey modificado) agrega descripción de abscesos a distancia, drenables o no por punción. 


\section{Método}

Tipo de estudio: Estudio descriptivo retrospectivo (cohorte), consideró más bien una serie de casos de pacientes hospitalizados en su centro entre los años 2009-2015 por DA en que la TC AP del ingreso mostró presencia de burbujas extraluminales pericolónicas y/o neumoperitoneo. Al respecto considero que faltó mencionar otros hallazgos como abscesos, líquido libre, etc., y tal vez definir y diferenciar el grupo de burbujas pericolónicas del neumoperitoneo a distancia, como lo hace el grupo de Alberto TitosGarcía que destaca en las Tablas, quienes consideraron aire a distancia cuando la burbuja se encontraba a más de $5 \mathrm{~cm}$ del colon afectado, buscando así un grupo potencialmente diferente en cuanto a riesgo, lo cual podría enriquecer el trabajo.

Los criterios de inclusión están bien enumerados, no así los de exclusión. Pudiera definirse con más detalle a qué se refiere estabilidad hemodinámica y dolor abdominal controlado, de manera de poder comparar en forma más precisa, pues serán estos mismos los que en parte definirán la falla del manejo conservador. Comento, además, que es posible que quien haya realizado la primera evaluación y definición de tratamiento en la Urgencia, sea un médico distinto a quien definió el tratamiento $\mathrm{y}$ seguimiento (como posiblemente ocurre en todos nuestros Servicios) y, por lo tanto, la evaluación inicial descrita por el especialista sea la de un paciente ya estabilizado.

La variable intervención es el manejo conservador, que queda claro. Los criterios de falla también están bien definidos. Al respecto menciona la "progresión imagenológica de la DPA“, pero da la impresión que no se hizo control de TC en todos los pacientes sino en forma selectiva, lo que me parece en todo caso razonable.

\section{Resultados} DA.

162 pacientes hospitalizados en el período por

Llama la atención que el 46,9\% (76) por DS 86 (43\%) por DC, y de estos 37 (43\%) correspondientes a DPA que fueron a manejo conservador.

Todos los pacientes, incluidos 8 de los 37 que tenían neumoperitoneo a distancia, fueron a tratamiento conservador.

El manejo conservador fue exitoso en 32 de los 37 casos, correspondiente al 86,5\%, muy bueno y concordante con cifras de otros trabajos publicados.

Debo mencionar eso sí, que quedó bien definido el fallo del tratamiento médico, pero no el éxito.

Se destaca un muy buen seguimiento y adherencia al tratamiento, completando todos el tratamien- to antibiótico indicado por 14 días, realizándose en diferido en todos una colonoscopía o enema baritado.

En el seguimiento a largo plazo se operaron 10 pacientes de los tratados exitosamente en forma conservadora, no explica por qué. En tanto 9 casos, también de tratamiento exitoso, presentaron síntomas en el futuro e incluso DA y, sin embargo, no fueron operados. ¿Había alguna característica distinta entre estos dos grupos? ¿Evolucionaron distinto? ¿Tuvieron un manejo distinto? ¿Qué afectó su calidad de vida para decidir su operación?

La morbilidad en los operados fue $0 \%$, sorprendentemente buena.

En cuanto a los pacientes en que falló el tratamiento médico $5(13,5 \%)$, resulta ser un grupo particular por cuanto son de mayor comorbilidad, uno finalmente era un cáncer de colon y no diverticular, siendo criterio de exclusión en algunos trabajos, y 2 pacientes de buena respuesta inicial y que reingresan por desarrollar colecciones intraabdominales, pero que fueron adecuadamente tratados por punción, no requiriendo cirugía de urgencia, estos casos en otros trabajos publicados no son considerados fracaso del tto médico.

\section{Discusión}

Los datos obtenidos en este estudio muestran que la mayoría de los pacientes con DPA pueden recibir un tratamiento no quirúrgico con una tasa de éxito del $86,5 \%$ y con un $28,1 \%$ de recurrencia, siendo todos susceptibles a recibir manejo médico con respuesta favorable.

El bajo $\mathrm{N}$ puede dificultar el análisis.

Pudiese haber enriquecido el trabajo, haber tratado de medir volumétricamente la cantidad de aire libre mediante TC de tal modo de lograr tal vez una caracterización de grupos de mayor o menor riesgo.

Pudiese ser un estudio a futuro.

\section{Conclusiones}

Afirma que "los pacientes tratados con éxito tienen un riesgo similar de recurrencia que los pacientes que presentan episodios sin complicaciones, por lo tanto, la indicación de sigmoidectomía electiva debería ser individualizada“.

Si bien comparto este criterio, no es una conclusión que puede ser derivada de su trabajo por cuanto de los 32 pacientes que se consideraron un éxito, 10 fueron operados en forma electiva, sin mencionar nada acerca de su historia y, por tanto, de la indicación quirúrgica. Lo más probable fue decisión de cada médico tratante de acuerdo a su visión del tema. 


\section{En resumen}

La Dra. Fernández nos presenta un trabajo muy interesante, de un tema actual que motiva a otros Centros a que expongamos resultados al respecto.

El carácter del trabajo, descriptivo retrospectivo, como son la mayoría de los publicados en la literatura internacional, y el bajo $\mathrm{N}$ limitan la extracción de conclusiones, pero sin duda marcan una ruta.

Es un gran esfuerzo por describir una serie de casos con este hallazgo que hasta hoy en día es de manejo controversial. La descripción es valiosa dado que nos muestra que es posible el manejo conservador en estos pacientes evitando la cirugía en gran parte de ellos.

Sr. Presidente, con mucho agrado doy por aceptado este trabajo de ingreso.

Dr. Rodrigo Miguieles $C$. 